DOI: $10.21608 / z v i z .2019 .28653$.

\title{
Economic Assessment of Various Levels of Protein and Energy in Pigeon Squabs Diet Mohamed A.E. Omar ${ }^{*}$, Fardos A.M. Hassan and Sara E. Shahin \\ Animal Wealth Development Department, Faculty of Veterinary Medicine, Zagazig University, 44511, Egypt
}

\begin{abstract}
This study was conducted to evaluate the effect of nine diets through the application of $3 \times 3$ randomized design which included three levels of energy $(2600,2800$ and $3000 \mathrm{Kcal} / \mathrm{kg}$ ) with 3 supplemental protein levels $(14,16$ and $18 \%)$ on growth performance, blood parameters, carcass yields and economic efficiency of Baladi pigeon squabs from 28 days old until 6 months of age (age of sexual maturity). A total of 135 pairs of squabs were randomly and independently allocated to the nine dietary treatments, each containing 15 pairs ( 3 replicate of 5 pairs). The diets were formulated in mash form fortified with vitamin and mineral premix. Moreover, feed and water were provided for ad-libitum consumption. The results showed that, squabs fed higher energy and crude protein diets $(3000 \mathrm{ME}+16 \% \mathrm{CP})$ had higher live body weight with a coincident significant decrease of the feed intake $(\mathrm{P}<0.0001)$. In addition, dressing $(\%)$, breast and thigh meat yield (\%) increased significantly with increasing levels of both energy and protein, where they hit the peak values in squabs fed on $3000 \mathrm{ME}+16 \% \mathrm{CP}$ diet. Furthermore, increasing energy levels did not affect serum proteins (albumin and globulin), urea and uric acid. However, it had a significant positive effect on serum lipids (cholesterol, triglyceride, high density lipoprotein and low density lipoprotein). In terms of economic analysis, $3000 \mathrm{ME}+16 \%$ $\mathrm{CP}$ diet revealed the lowest cost per $\mathrm{Kg}$ live weight of birds $81.87 \mathrm{~L}$.E per pair, along with the highest economic return 110.3 L.E per pair and net profit 10.43 L.E per pair. In conclusion, squabs can efficiently utilize diet containing high energy $(3000 \mathrm{Kcal} / \mathrm{kg})$ and crude protein $(16 \%)$ diets, consequently, it can be used to optimize their growth performance and maintain the maximum economic return.
\end{abstract}

Key words: Pigeons growth performance, Crude Protein, Metabolic Energy, Economics of squabs.

\section{Introduction}

Domestic pigeons (Columba livia) are multi-purpose birds, as they can be used for meat production (because of their powerful breast muscles and high growth rate), sports (racing) and as experimental animals [1]. However, in Egypt, especially in the rural areas, pigeons are kept mainly for meat production which plays an important role in the profitability of both small-scale and commercial farms. Compared with the other poultry, pigeons are deemed economic meat producers because of the lower costs of their feed, caring, housing and capital investment. Moreover, it has a relatively long productive life and short reproductive cycle besides its high immunity against diseases. Meat of the baby pigeon (squabs at the age of 28-30 days) has a high nutritive value as it is rich in protein, minerals and vitamins. In addition, it is very delicate, lean (low cholesterol), easily digested and considered as fancy meat because of its taste and delicacy [2,3]. Until now, there is insufficient information about nutrient requirements or growth curves of pigeons, as the National Research Council (NRC) has not provided any standards or guidelines $[4,5]$.

Squabs are fed by their parents (both male and female) with "crop milk" in the first 5-7 days of life; therefore, the feeding system of pigeons differs markedly from of different poultry species [6]. Pigeon's diet consists mainly of whole grain which could supply enough protein, carbohydrates and fat, however it is scanty of necessary vitamins, macro and micro nutrients. Metabolizable energy (ME) and crude protein (CP) are the costliest components of the pigeon's diet as they constituted about $85 \%$ of the gross feed costs. Moreover, they are significantly implemented in their growing and laying performance. Therefore, their levels in the diets should be optimized cautiously during ration formulation, in order to get the least cost with maximum production as well [7]. Some 
studies were conducted to evaluate the impact of different dietary CP 12 to $18 \%$ and ME content (2600, 2800 and $3000 \mathrm{Kcal}$ $\mathrm{ME} / \mathrm{kg}$ ) separately on the productivity of pigeons $[1,8]$. Whereas, the present study aimed to evaluate the diets formulated by combing three levels of CP $(14,16$ and $18 \%)$ and three levels of $\mathrm{ME}(2600,2800$ and $3000 \mathrm{Kcal} / \mathrm{kg}$ diet) on the growth performance, carcass traits serum parameters and economic efficiency of Baladi growing squabs during $1^{\text {st }}$ six month and before laying under Egyptian conditions.

\section{Material and Methods}

This study was conducted at especially constructed farm, Elkenayat, Zagazig, Sharkia Governorate, Egypt. All the experimental methods were done according to the Committee of Community Service and Environment Development, Zagazig University, Egypt. Squabs were cared for using husbandry guidelines and standard operating procedures derived from Department of Avian and Rabbit Medicine, Faculty of Veterinary Medicine, Zagazig University, Zagazig, Egypt. During the period from $1^{\text {st }}$ February until the end of June 2017 with a view to study the efficiency of feeding different metabolic energy and protein levels in diets on productive, physiological and economic performance of growing squabs.

\section{Birds, design, housing and experimental diets}

A total number of 135 pairs of Baladi pigeon squabs (aged 28-30 days), obtained from three small scale pigeon hatcheries were allocated to nine dietary treatments, each containing 3 replicates with 5 pairs per each. A $3 \times 3$ factorial experiment using 3 dietary ME levels (2600, 2800 and 3000 $\mathrm{Kcal} / \mathrm{kg}$ ) and 3 supplemental CP levels (14, 16 and $18 \%$ ) was conducted to study the effects of dietary energy levels and CP levels. Squabs in all treatments were fed $a d$ libitum. The squabs were housed in an environmentally controlled home with each pair was kept in individual pigeon breeding cage (width $100 \times$ length $70 \times$ height 40 $\mathrm{cm})$, prepared to keep pigeon pair separately. The fronts of the cage batteries were modified to suspend feed and water. All squabs were treated with piprazin drugs during the quarantine period to ensure eliminating of any parasites that can affect the performance. The lighting schedule program used through the experimental period was natural daylight and artificial light (60-watt electric bulb intensity), provide 14 light vs 10 dark and the mean daily temperature was $25 \pm 4 \mathrm{C}^{\circ}$ [9]. Diets were formulated as mash form consists of 3 protein levels (14-16 and 18\%) and 3 energy levels were used (2600, 2800, and $3000 \mathrm{ME}$ $\mathrm{Kcal} / \mathrm{kg}$ of diet). The components of the diets were showed in Table (1).

\section{Experimental parameters}

\section{Growth performance measurements}

Through the experiment, the following measurements were recorded: initial weight at 28 day of age, average final weight at 180 day of age, daily feed intake per pair, total feed intake per pair (during 5 months).

\section{Carcass traits measurements}

At 6 months of age, 6 birds (two birds per replicate) group were taken randomly for carcass analysis. The squabs were weighed, sacrificed and left to bleed for 3 minutes and then scalded at $60^{\circ} \mathrm{C}$ for 1 minute. After that they were de-feathered and manually eviscerated to study carcass characteristics. Each carcass was weighed to record the dressing carcass weights and all values were calculated as percentages of live body weights.

\section{Blood Parameters}

On a random basis, blood samples were collected from 6 squabs per group (2 birds per replicate) at slaughtering. Triglycerides (TG), total cholesterol (TC), high density lipoprotein (HDL), low density lipoprotein (LDL), total plasma proteins (TP), albumin (ALB), globulin (GLB), urea and uric acid (UA) were determined calorimetrically by using the commercial kits (ELITech SEEPPIM S.A.S. Zone industrielle - 61500 France). 


\section{Economic analysis}

At the end of experiment, the following indices were calculated per pair: feed cost (FC), total costs (TC), total return (NR), net profit (NP) and economic efficiency (EE).

\section{Statistical Analysis}

Data were analyzed as a $3 \times 3$ factorial arrangement in a randomized complete block design to find the main effects of 3 levels of CP and 3 levels of ME and their corresponding interaction effects using the GLM procedure and means were separated using Bonferroni's adjusted multiple comparison. Analysis was done using Statistical Package for Social Sciences version 22.0 (IBM Corp., Armonk, NY, USA) and Graphpad PRISM 7.0 (San Diego, CA) software. Results were reported in means \pm SEM (Standard Error of Mean). The value of $\mathrm{P}<0.05$ was used to indicate statistical significance. Cage (pair) served as assessment unit for all measurements for the statistical analysis of live performance. Differences were considered significant at $\mathrm{P}$
$<0.05$. The effect of replicate and hatchery location was incorporated in the initial model to determine if significant differences exist.

\section{Results}

\section{Growth performance}

The effects of different levels of dietary $\mathrm{ME}$ and $\mathrm{CP}$ on both BW and FI were showed in Table 2. From 2 to 5 months of the experiment, BW and FI of squabs were significantly improved by increasing dietary levels of both ME and CP with significant $(\mathrm{P}<0.0001) \mathrm{ME} \times \mathrm{CP}$ interaction effect. In particular, when $3000 \mathrm{Kcal} / \mathrm{Kg} \mathrm{ME}+16 \%$ $\mathrm{CP}$ diets were fed to the squabs, higher $\mathrm{BW}$ was observed with a coincident decrease in FI. However, further increasing of CP level in $3000 \mathrm{Kcal} / \mathrm{Kg} \mathrm{ME}$ diets did not lead to a significant change in growth parameters. The mortality rate was low $13.3 \%$ with no signs of either bacterial or viral infection, however higher losses were reported in squabs fed $18 \% \mathrm{CP}$ diets. 
Table 1: Ingredients and calculated composition $(\%)$ of the experimental diets

\begin{tabular}{|c|c|c|c|c|c|c|c|c|c|}
\hline \multicolumn{10}{|l|}{ Items } \\
\hline \multirow{2}{*}{$\begin{array}{l}\mathrm{CP}(\%)^{1} \\
\mathrm{ME}(\mathrm{Kcal})^{2}\end{array}$} & \multicolumn{3}{|c|}{$14 \%$} & \multicolumn{3}{|c|}{$16 \%$} & \multicolumn{3}{|c|}{$18 \%$} \\
\hline & 2600 & 2800 & 3000 & 2600 & 2800 & 3000 & 2600 & 2800 & 3000 \\
\hline \multicolumn{10}{|l|}{ Ingredients } \\
\hline yellow corn (kg) & 11 & 23 & 24.7 & - & 8 & 3.8 & - & - & - \\
\hline Wheat (kg) & 18 & 26 & 30 & 29 & 32 & 35.5 & 30.7 & 21 & 10 \\
\hline Millet (kg) & 35.7 & 12 & - & 22.2 & 5 & - & - & - & - \\
\hline Horse beans $(\mathrm{kg})$ & 31 & 33.7 & 37 & 44 & 48.2 & 50.4 & 64 & 69.5 & 76 \\
\hline Soybean oil (kg) & - & 1 & 4 & 0.5 & 2.5 & 6 & 1 & 5.2 & 9.7 \\
\hline Calcium carbonate $(\mathrm{kg})$ & 1.5 & 1.5 & 1.5 & 1.5 & 1.5 & 1.5 & 1.5 & 1.5 & 1.5 \\
\hline Calcium dibasic phosphate $(\mathrm{kg})$ & 2 & 2 & 2 & 2 & 2 & 2 & 2 & 2 & 2 \\
\hline Common salt $(\mathrm{kg})$ & 0.3 & 0.3 & 0.3 & 0.3 & 0.3 & 0.3 & 0.3 & 0.3 & 0.3 \\
\hline $\operatorname{Premix}^{3}(\mathrm{~kg})$ & 0.3 & 0.3 & 0.3 & 0.3 & 0.3 & 0.3 & 0.3 & 0.3 & 0.3 \\
\hline Antimycotoxin (kg) & 0.1 & 0.1 & 0.1 & 0.1 & 0.1 & 0.1 & 0.1 & 0.1 & 0.1 \\
\hline Anticoccidial $(\mathrm{kg})$ & 0.1 & 0.1 & 0.1 & 0.1 & 0.1 & 0.1 & 0.1 & 0.1 & 0.1 \\
\hline \multicolumn{10}{|l|}{ Calculated composition } \\
\hline $\mathrm{ME}, \mathrm{Kcal} / \mathrm{Kg}$ & 2645.58 & 2820.01 & 3026.55 & 2623.45 & 2804.21 & 3001.82 & 2613.32 & 2810.05 & 3016.4 \\
\hline $\mathrm{CP} \%$ & 14.32 & 14.07 & 14.02 & 16.11 & 16 & 16.01 & 18.07 & 18.02 & 18.02 \\
\hline $\mathrm{EE} \%{ }^{4}$ & 2.6 & 2.19 & 1.86 & 1.98 & 1.65 & 1.37 & 1.36 & 1.29 & 1.21 \\
\hline $\mathrm{CF} \%^{5}$ & 4.02 & 4.01 & 4.03 & 4.62 & 4.66 & 4.68 & 5.4 & 5.5 & 5.62 \\
\hline $\mathrm{Ca} \%{ }^{6}$ & 1.16 & 1.16 & 1.16 & 1.16 & 1.17 & 1.17 & 1.18 & 1.18 & 1.18 \\
\hline Available phosphorus $\%$ & 0.43 & 0.42 & 0.41 & 0.42 & 0.4 & 0.4 & 0.39 & 0.38 & 0.37 \\
\hline Lysine $\%$ & 0.72 & 0.72 & 0.74 & 0.87 & 0.89 & 0.9 & 1.08 & 1.12 & 1.18 \\
\hline Methionine \% & 0.21 & 0.2 & 0.19 & 0.21 & 0.2 & 0.19 & 0.2 & 0.18 & 0.16 \\
\hline
\end{tabular}

${ }^{1} \mathrm{CP}$ : crude protein ${ }^{2} \mathrm{ME}$ : metabolizable energy; ${ }^{3}$ Muvco premix: Each $2.5 \mathrm{~kg}$ contain vit. A (10, $\left.000000 \mathrm{IU}\right)$,vit. D3 (2, 000000 IU), vit. E ( 10 g),vit k3 (1000 mg), vit. B1 $(1000 \mathrm{mg})$, vit. B2( $5 \mathrm{~g})$, vit.B6 (1.5 g), pantothenic acid( $10 \mathrm{~g})$, vit. B12 (10 mg ), niacin( $30 \mathrm{~g})$, folic acid (1000 mg ), biotin(50 g), fe (30 g) , Mn (60 g), Cu (4 g), I (300 mg), $\mathrm{Co}(100 \mathrm{mg}), \mathrm{Se}(100 \mathrm{mg})$ and $\mathrm{Zn}(50 \mathrm{~g}),{ }^{4} \mathrm{EE}$ : ether extract; ${ }^{5} \mathrm{CF}$ : crude fiber; ${ }^{6} \mathrm{Ca}$ : calcium. 
Table 2: Body weight (g) and daily feed intake* of squabs fed diets with different ME and CP levels

\begin{tabular}{|c|c|c|c|c|c|c|c|c|c|c|c|c|c|}
\hline \multirow[t]{2}{*}{$M E^{I}$} & \multirow[t]{2}{*}{$\mathbf{C P}^{2}$} & \multirow{2}{*}{$\begin{array}{c}\text { Initial } \\
\text { (28 days) }\end{array}$} & \multicolumn{2}{|c|}{2 Month } & \multicolumn{2}{|c|}{3 Month } & \multicolumn{2}{|c|}{4 Month } & \multicolumn{2}{|c|}{5 Month } & \multicolumn{2}{|c|}{6 Month } & \multirow{2}{*}{ TFI $^{4}$} \\
\hline & & & Body weight & DFI $^{3}$ & Body weight & DFI & $\begin{array}{c}\text { Body } \\
\text { weight }\end{array}$ & DFI & $\begin{array}{c}\text { Body } \\
\text { weight }\end{array}$ & DFI & $\begin{array}{c}\text { Body } \\
\text { weight }\end{array}$ & DFI & \\
\hline \multirow{6}{*}{2600} & \multirow{2}{*}{14} & 270.01 & 286.55 & 26.8 & 290.63 & 38.01 & 295.83 & 48.41 & 300.62 & 54.8 & 311.4 & 61.99 & 6900.3 \\
\hline & & \pm 13.17 & \pm 11.92 & \pm 0.72 & $\pm 12.08^{\mathrm{e}}$ & $\pm 0.55^{\mathrm{a}}$ & $\pm 7.37^{\mathrm{d}}$ & $\pm 2.08^{\mathrm{ab}}$ & $\pm 9.53^{\mathrm{d}}$ & $\pm 1.82^{\mathrm{a}}$ & $\pm 2.51^{\mathrm{e}}$ & $\pm 1.43^{\mathrm{a}}$ & $\pm 12.01^{\mathrm{a}}$ \\
\hline & \multirow{2}{*}{16} & 267.97 & 283.29 & 25.93 & 294.14 & 38.98 & 303.13 & 48.64 & 313.42 & 53.09 & 322.8 & 65.32 & 6958.8 \\
\hline & & \pm 11.97 & \pm 8.94 & \pm 0.49 & $\pm 9.31^{\mathrm{d}}$ & $\pm 0.75^{\mathrm{a}}$ & $\pm 6.83^{c}$ & $\pm 2.55^{\mathrm{ab}}$ & $\pm 7.27^{\mathrm{c}}$ & $\pm 1.52^{\mathrm{ab}}$ & $\pm 9.93^{\mathrm{d}}$ & $\pm 1.3^{\mathrm{a}}$ & $\pm 25.16^{\mathrm{a}}$ \\
\hline & \multirow{2}{*}{18} & 263.80 & 279.96 & 26.32 & 293.01 & 33.92 & 307.36 & 51.07 & 316.64 & 54.62 & 323.71 & 64.29 & 6906.6 \\
\hline & & \pm 13.8 & \pm 14.36 & \pm 0.88 & $\pm 10.98^{\mathrm{d}}$ & $\pm 1.22^{\mathrm{ab}}$ & $\pm 12.33^{\mathrm{c}}$ & $\pm 2.27^{\mathrm{a}}$ & $\pm 11.85^{\mathrm{c}}$ & $\pm 1.53^{\mathrm{a}}$ & $\pm 11.88^{\mathrm{d}}$ & $\pm 0.9^{\mathrm{a}}$ & $\pm 18.39^{\mathrm{a}}$ \\
\hline \multirow{6}{*}{2800} & \multirow{2}{*}{14} & 260.21 & 288.96 & 25.81 & 304.39 & 32.53 & 313.2 & 46.19 & 321.8 & 49.45 & 336.1 & 54.12 & 6243 \\
\hline & & \pm 5.37 & \pm 10.67 & \pm 0.7 & $\pm 8.68^{\mathrm{c}}$ & $\pm 0.83^{\mathrm{ab}}$ & $\pm 9.77^{\mathrm{c}}$ & $\pm 2.22^{\mathrm{ab}}$ & $\pm 8.07^{\mathrm{c}}$ & $\pm 1.19^{\mathrm{ab}}$ & $\pm 11.67^{\mathrm{c}}$ & $\pm 1.08^{\mathrm{b}}$ & $\pm 11.91^{\mathrm{ab}}$ \\
\hline & \multirow{2}{*}{16} & 279.66 & 296.26 & 24.83 & 311.04 & 36.68 & 325.87 & 38.45 & 339.05 & 47.24 & 349.28 & 52.94 & 6004.2 \\
\hline & & \pm 4.67 & \pm 5.81 & \pm 1.19 & $\pm 12.21^{\mathrm{b}}$ & $\pm 1.38^{\mathrm{ab}}$ & $\pm 8.28^{\mathrm{b}}$ & $\pm 1.1^{\mathrm{b}}$ & $\pm 8.13^{\mathrm{b}}$ & $\pm 1.56^{\mathrm{b}}$ & $\pm 7.99^{\mathrm{b}}$ & $\pm 1.59^{\mathrm{b}}$ & $\pm 22.4^{b}$ \\
\hline & \multirow{2}{*}{18} & 273.57 & 293.45 & 25.21 & 314.21 & 36.99 & 327.62 & 40.46 & 338.1 & 47.72 & 348.72 & 53.77 & 6124.5 \\
\hline & & \pm 9.56 & \pm 4.81 & \pm 0.59 & $\pm 6.52^{\mathrm{b}}$ & $\pm 1.44^{\mathrm{ab}}$ & $\pm 11.08^{\mathrm{b}}$ & $\pm 1.02^{\mathrm{b}}$ & $\pm 12.24^{\mathrm{b}}$ & $\pm 1.49^{\mathrm{b}}$ & $\pm 6.9^{\mathrm{b}}$ & $\pm 1.81^{\mathrm{b}}$ & $\pm 14.11^{\mathrm{b}}$ \\
\hline \multirow{6}{*}{3000} & \multirow{2}{*}{14} & 275.09 & 305.21 & 25.19 & 318.86 & 28.24 & 323.49 & 41.1 & 341.9 & 41.08 & 358.18 & 48.66 & 5528.1 \\
\hline & & \pm 6.4 & \pm 6.14 & \pm 0.87 & $\pm 8.35^{\mathrm{ab}}$ & $\pm 2.07^{\mathrm{c}}$ & $\pm 7.7^{\mathrm{b}}$ & $\pm 0.93^{\mathrm{b}}$ & $\pm 8.50^{\mathrm{b}}$ & $\pm 1.01^{\mathrm{c}}$ & $\pm 7.69^{\mathrm{b}}$ & $\pm 0.67^{\mathrm{bc}}$ & $\pm 10.91^{\mathrm{c}}$ \\
\hline & \multirow{2}{*}{16} & 280.61 & 309.63 & 25.36 & 321.08 & 26.27 & 341.8 & 32.44 & 379.33 & 39.08 & 407.27 & 41.4 & 4936.5 \\
\hline & & \pm 4.79 & \pm 7.91 & \pm 0.98 & $\pm 11.99^{\mathrm{ab}}$ & $\pm 0.97^{\mathrm{c}}$ & $\pm 9.19^{\mathrm{a}}$ & $\pm 1.38^{\mathrm{c}}$ & $\pm 10.26^{\mathrm{a}}$ & $\pm 0.94^{\mathrm{c}}$ & $\pm 12.7^{\mathrm{a}}$ & $\pm 1.31^{\mathrm{d}}$ & $\pm 16.71^{\mathrm{d}}$ \\
\hline & \multirow{2}{*}{18} & 271.54 & 301.01 & 25.02 & 325.85 & 29.66 & 351.09 & 39.61 & 372.24 & 39.86 & 411.6 & 44.31 & 5353.8 \\
\hline & & \pm 5.8 & \pm 7.17 & \pm 1.02 & $\pm 12.1^{\mathrm{a}}$ & $\pm 1.02^{\mathrm{c}}$ & $\pm 6.06^{\mathrm{a}}$ & $\pm 1^{\mathrm{b}}$ & $\pm 8.11^{\mathrm{a}}$ & $\pm 0.74^{\mathrm{c}}$ & $\pm 9.65^{\mathrm{a}}$ & $\pm 1.35^{\mathrm{c}}$ & $\pm 19.51^{\mathrm{c}}$ \\
\hline \multicolumn{14}{|c|}{$P$-values } \\
\hline \multicolumn{2}{|l|}{$M E$} & 0.661 & 0.329 & 0.283 & 0.009 & 0.003 & $<0.0001$ & $<0.0001$ & $<0.0001$ & $<0.0001$ & $<0.0001$ & $<0.0001$ & 0.253 \\
\hline \multicolumn{2}{|l|}{$C P$} & 0.848 & 0.961 & 0.137 & $<0.0001$ & $<0.0001$ & $<0.0001$ & 0.619 & $<0.0001$ & 0.323 & $<0.0001$ & 0.455 & $<0.0001$ \\
\hline \multicolumn{2}{|c|}{$M E \times C P$} & 0.629 & 0.414 & 0.544 & $<0.0001$ & $<0.0001$ & $<0.0001$ & $<0.0001$ & $<0.0001$ & $<0.0001$ & $<0.0001$ & $<0.0001$ & $<0.0001$ \\
\hline
\end{tabular}

Daily feed intake per pair of squabs; ${ }^{1} \mathrm{ME}$ : metabolizable energy; ${ }^{2} \mathrm{CP}$ : crude protein; ${ }^{3} \mathrm{DFI}$ : daily feed intake; ${ }^{4} \mathrm{TFI}$ : total feed intake.

Means within the same column carrying different superscripts were significantly different at $\mathrm{P}<0.05$ based on Bonferroni multiple comparison test. 
Table 3. Carcass traits and relative organ weights of squabs fed diets with different ME and protein levels

\begin{tabular}{lcccccccc}
\hline $\mathrm{ME}^{1}$ & $\mathrm{CP}^{2}$ & $\begin{array}{c}\text { Dressing } \\
(\%)\end{array}$ & $\begin{array}{c}\text { Breast meat } \\
(\%)\end{array}$ & $\begin{array}{c}\text { Thigh meat } \\
(\%)\end{array}$ & $\begin{array}{c}\text { Liver } \\
\%\end{array}$ & $\begin{array}{c}\text { Heart } \\
\%\end{array}$ & $\begin{array}{c}\text { Gizzard } \\
\%\end{array}$ & $\begin{array}{c}\text { Small intestine } \\
\%\end{array}$ \\
\hline 2600 & 14 & $55.2 \pm 7.31^{\mathrm{d}}$ & $13.2 \pm 3.84^{\mathrm{c}}$ & $4.47 \pm 2.11^{\mathrm{c}}$ & $2.31 \pm 0.29$ & $1.33 \pm 0.27$ & $1.92 \pm 0.27$ & $0.19 \pm 0.01$ \\
& 16 & $60.5 \pm 5.51^{\mathrm{c}}$ & $12.94 \pm 4.52^{\mathrm{c}}$ & $4.68 \pm 2.06^{\mathrm{c}}$ & $2.35 \pm 0.4$ & $1.39 \pm 0.32$ & $1.97 \pm 0.25$ & $0.41 \pm 0.05$ \\
& 18 & $61 \pm 8.39^{\mathrm{c}}$ & $13.7 \pm 3.89^{\mathrm{c}}$ & $5.16 \pm 2.4^{\mathrm{b}}$ & $2.41 \pm 0.46$ & $1.4 \pm 0.29$ & $1.98 \pm 0.3$ & $0.46 \pm 0.1$ \\
2800 & 14 & $57.5 \pm 7.89^{\mathrm{d}}$ & $15.20 \pm 2.29^{\mathrm{b}}$ & $5.34 \pm 5.72^{\mathrm{b}}$ & $2.24 \pm 0.54$ & $1.37 \pm 0.23$ & $1.93 \pm 0.29$ & $0.23 \pm 0.09$ \\
& 16 & $64.6 \pm 6.89^{\mathrm{b}}$ & $14.91 \pm 2.8^{\mathrm{b}}$ & $5.58 \pm 5.54^{\mathrm{b}}$ & $2.50 \pm 0.33$ & $1.41 \pm 0.37$ & $1.99 \pm 0.34$ & $0.45 \pm 0.1$ \\
& 18 & $66.8 \pm 5.85^{\mathrm{b}}$ & $16.1 \pm 4.64^{\mathrm{b}}$ & $5.64 \pm 2.48^{\mathrm{b}}$ & $2.41 \pm 0.41$ & $1.49 \pm 0.39$ & $2.01 \pm 0.45$ & $0.39 \pm 0.06$ \\
3000 & 14 & $56.8 \pm 5.97^{\mathrm{d}}$ & $16.5 \pm 3.34^{\mathrm{b}}$ & $5.11 \pm 3.47^{\mathrm{b}}$ & $2.33 \pm 0.37$ & $1.47 \pm 0.28$ & $1.96 \pm 0.36$ & $0.48 \pm 0.04$ \\
& 16 & $70.1 \pm 7.02^{\mathrm{a}}$ & $18.2 \pm 3.56^{\mathrm{a}}$ & $6.96 \pm 3.52^{\mathrm{a}}$ & $2.52 \pm 0.52$ & $1.50 \pm 0.34$ & $2.1 \pm 0.47$ & $0.3 \pm 0.08$ \\
$\mathrm{P}-\mathrm{values}$ & 18 & $69.3 \pm 6.1^{\mathrm{a}}$ & $18.9 \pm 4.31^{\mathrm{a}}$ & $6.51 \pm 2.69^{\mathrm{a}}$ & $2.66 \pm 0.33$ & $1.53 \pm 0.3$ & $2.34 \pm 0.39$ & $0.53 \pm 0.05$ \\
$\mathrm{ME}$ & & & & & & & \\
$\mathrm{CP}$ & $<0.0001$ & $<0.0001$ & $<0.0001$ & 0.936 & 0.663 & 0.317 & 0.645 \\
$\mathrm{ME} \times \mathrm{CP}$ & $<0.0001$ & $<0.0001$ & $<0.0001$ & 0.857 & 0.248 & 0.486 & 0.629 \\
\hline
\end{tabular}

${ }^{1} \mathrm{ME}:$ metabolizable energy; ${ }^{2} \mathrm{CP}$ : crude protein.

Means within the same column carrying different superscripts were significantly different at $\mathrm{P}<0.05$ based on Bonferroni multiple comparison test.

\section{Carcass traits and relative organ weights}

The effects of different levels of dietary $\mathrm{ME}$ and $\mathrm{CP}$ on carcass traits and relative organ weights were showed in Table 3. Highest dressing \%, breast and thigh meat \% were obtained from squabs fed on 3000 $\mathrm{Kcal} / \mathrm{Kg} \mathrm{ME}+16 \% \mathrm{CP}$ diets. On the other hand, there were no $\mathrm{ME}, \mathrm{CP}$ and $\mathrm{ME} \times \mathrm{CP}$ interaction effects on the relative weights of the other body organs (Liver \%, Heart \%, Gizzard\% and Small intestine \%).

\section{Biochemical analysis}

The effects of different levels of dietary $\mathrm{ME}$ and $\mathrm{CP}$ on blood parameters of squabs at $6^{\text {th }}$ month of age were presented in Table 4. High protein diets resulted in higher serum proteins (TP, ALB and GLB) along with significant increase in the concentrations of urea and uric acid regardless of the dietary ME content. In contrast, higher TC, TG, HDL and LDL were observed in serum samples obtained from squabs fed on high ME diets.

\section{Economic analysis}

The effects of different levels of dietary $\mathrm{CP}$ and $\mathrm{ME}$ on the economic indices were showed in Table 5. Feed cost and total cost decreased with increasing of dietary energy and protein levels. The higher values of TC were recorded in squabs fed $3000 \mathrm{Kcal} \mathrm{ME}$ $+16 \%$ CP diet. Furthermore, TR, NP and EE the highest values for squabs fed diets containing 3000 Kcal ME $+16 \% \quad \mathrm{CP}$ combinations compared with other groups. 
Table 4: Serum biochemical parameters of squabs fed diets with different ME and CP levels

\begin{tabular}{|c|c|c|c|c|c|c|c|c|c|c|}
\hline $\mathrm{ME}^{1}$ & $\mathbf{C P}^{2}$ & $\begin{array}{l}\mathbf{A L B}^{3} \\
(\mathrm{~g} / \mathbf{d L})\end{array}$ & $\operatorname{GLB}^{4}(\mathrm{~g} / \mathrm{dL})$ & $\begin{array}{c}\mathbf{T P}^{5} \\
(\mathrm{~g} / \mathrm{dL})\end{array}$ & $\begin{array}{c}\text { Urea } \\
(\mathrm{mg} / \mathrm{dL})\end{array}$ & $\mathrm{UA}^{6}(\mathrm{mg} / \mathrm{dL})$ & $\begin{array}{c}\mathrm{TC}^{7} \\
(\mathrm{mg} / \mathrm{dL})\end{array}$ & $\begin{array}{c}\mathbf{T G}^{\mathbf{8}} \\
(\mathbf{m g} / \mathbf{d L})\end{array}$ & $\begin{array}{c}\text { HDL }^{9} \\
(\mathrm{mg} / \mathrm{dL})\end{array}$ & $\begin{array}{c}\mathrm{LDL}^{10} \\
(\mathrm{mg} / \mathrm{dL})\end{array}$ \\
\hline \multirow{6}{*}{2600} & \multirow{2}{*}{14} & 1.09 & 1.98 & 3.08 & 2.71 & 4.11 & 136.83 & 113.93 & 40.9 & 71.57 \\
\hline & & $\pm 0.11^{\mathrm{c}}$ & $\pm 0.4^{\mathrm{c}}$ & $\pm 0.33^{c}$ & $\pm 0.25^{\mathrm{c}}$ & $\pm 0.14^{\mathrm{b}}$ & $\pm 7.43^{c}$ & $\pm 3.25^{c}$ & $\pm 2.82^{\mathrm{c}}$ & $\pm 2.22^{\mathrm{c}}$ \\
\hline & \multirow{2}{*}{16} & 2.24 & 2.83 & 5.07 & 4.12 & 5.16 & 140.13 & 115.47 & 37.07 & 72.67 \\
\hline & & $\pm 0.13^{b}$ & $\pm 0.12^{\mathrm{b}}$ & $\pm 0.18^{b}$ & $\pm 0.09^{b}$ & $\pm 0.17^{\mathrm{a}}$ & $\pm 4.89^{c}$ & $\pm 8.66^{c}$ & $\pm 1.99^{c}$ & $\pm 3.58^{c}$ \\
\hline & \multirow{2}{*}{18} & 2.91 & 4.13 & 7.04 & 5.1 & 6.45 & 150.2 & 107.1 & 40.73 & 69.07 \\
\hline & & $\pm 0.09^{b}$ & $\pm 0.25^{\mathrm{a}}$ & $\pm 0.35^{\mathrm{a}}$ & $\pm 0.1^{\mathrm{a}}$ & $\pm 0.32^{\mathrm{a}}$ & $\pm 2.83^{c}$ & $\pm 7.05^{c}$ & $\pm 2.07^{\mathrm{c}}$ & $\pm 4.84^{c}$ \\
\hline \multirow{6}{*}{2800} & \multirow{2}{*}{14} & 1.06 & 1.9 & 2.96 & 2.73 & 3.9 & 164.3 & 141.4 & 49.33 & 78.5 \\
\hline & & $\pm 0.13^{c}$ & $\pm 0.24^{\mathrm{c}}$ & $\pm 0.13^{\mathrm{c}}$ & $\pm 0.12^{c}$ & $\pm 0.11^{\mathrm{b}}$ & $\pm 3.02^{\mathrm{b}}$ & $\pm 2.75^{b}$ & $\pm 4.66^{\mathrm{b}}$ & $\pm 2.12^{b}$ \\
\hline & \multirow{2}{*}{16} & 2.26 & 2.88 & 5.14 & 3.81 & 5.26 & 166.4 & 155.57 & 52.17 & 86.77 \\
\hline & & $\pm 0.22^{\mathrm{b}}$ & $\pm 0.16^{\mathrm{b}}$ & $\pm 0.13^{b}$ & $\pm 0.15^{b}$ & $\pm 0.27^{\mathrm{a}}$ & \pm 5.92 & $\pm 6.58^{b}$ & $\pm 2.83^{b}$ & $\pm 2.19^{\mathrm{b}}$ \\
\hline & \multirow{2}{*}{18} & 3.38 & 3.95 & 7.34 & 5.66 & 6.88 & 169.77 & 153.87 & 53.33 & 81.73 \\
\hline & & $\pm 0.22^{\mathrm{a}}$ & $\pm 0.27^{\mathrm{a}}$ & $\pm 0.43^{\mathrm{a}}$ & $\pm 0.41^{\mathrm{a}}$ & $\pm 0.11^{\mathrm{a}}$ & $\pm 2.31^{\mathrm{b}}$ & $\pm 8.55^{b}$ & $\pm 2.39^{\mathrm{b}}$ & $\pm 2.87^{\mathrm{b}}$ \\
\hline \multirow{6}{*}{3000} & \multirow{2}{*}{14} & 1.32 & 1.76 & 3.08 & 2.45 & 3.79 & 181.93 & 174.43 & 73.8 & 93.93 \\
\hline & & $\pm 0.17^{\mathrm{c}}$ & $\pm 0.29^{c}$ & $\pm 0.44^{\mathrm{c}}$ & $\pm 0.07^{\mathrm{c}}$ & $\pm 0.28^{\mathrm{b}}$ & $\pm 1.53^{\mathrm{a}}$ & $\pm 5.72^{\mathrm{a}}$ & $\pm 2.4^{\mathrm{a}}$ & $\pm 2.5^{\mathrm{a}}$ \\
\hline & \multirow{2}{*}{16} & 2.21 & 2.94 & 5.15 & 3.93 & 4.96 & 179.93 & 175.2 & 70.53 & 101.33 \\
\hline & & $\pm 0.18^{b}$ & $\pm 0.17^{\mathrm{b}}$ & $\pm 0.31^{\mathrm{b}}$ & $\pm 0.14^{\mathrm{b}}$ & $\pm 0.22^{\mathrm{ab}}$ & $\pm 2.03^{\mathrm{a}}$ & $\pm 4.82^{\mathrm{a}}$ & $\pm 2.94^{\mathrm{a}}$ & $\pm 3.8^{\mathrm{a}}$ \\
\hline & \multirow{2}{*}{18} & 3.43 & 4.35 & 7.78 & 5.88 & 6.61 & 184.03 & 170.17 & 77.53 & 98.47 \\
\hline & & $\pm 0.27^{\mathrm{a}}$ & $\pm 0.27^{\mathrm{a}}$ & $\pm 0.2^{\mathrm{a}}$ & $\pm 0.45^{\mathrm{a}}$ & $\pm 0.37^{\mathrm{a}}$ & $\pm 5.45^{\mathrm{a}}$ & $\pm 2.66^{\mathrm{a}}$ & $\pm 0.54^{\mathrm{a}}$ & $\pm 2.71^{\mathrm{a}}$ \\
\hline \multicolumn{11}{|l|}{ P-values } \\
\hline \multicolumn{2}{|l|}{ ME } & 0.550 & 0.495 & 0.261 & 0.344 & 0.566 & $<0.0001$ & $<0.0001$ & $<0.0001$ & $<0.0001$ \\
\hline \multicolumn{2}{|l|}{$\mathrm{CP}$} & $<0.0001$ & 0.005 & $<0.0001$ & 0.003 & $<0.0001$ & 0.341 & 0.557 & 0.469 & 0.753 \\
\hline \multicolumn{2}{|l|}{$\mathrm{ME} \times \mathrm{CP}$} & $<0.0001$ & $<0.0001$ & $<0.0001$ & $<0.0001$ & $<0.0001$ & $<0.0001$ & $<0.0001$ & $<0.0001$ & $<0.0001$ \\
\hline
\end{tabular}

${ }^{1} \mathrm{ME}$ : metabolizable energy; ${ }^{2} \mathrm{CP}$ : crude protein; ${ }^{3} \mathrm{ALB}$ : albumin; ${ }^{4} \mathrm{GLB}$ : globulin; ${ }^{5} \mathrm{TP}$ : total proteins; ${ }^{6} \mathrm{UA}$ : uric acid; ${ }^{7} \mathrm{TC}:$ total cholesterol; ${ }^{8} \mathrm{TG}:$ Triglycerides; ${ }^{9} \mathrm{HDL}:$ high density lipoprotein;

${ }^{10}$ LDL: low density lipoprotein; Means within the same column carrying different superscripts were significantly different at $\mathrm{P}<0.05$ based on Bonferroni multiple comparison test 
Table 5: The effects of different levels of dietary ME and CP on the economic indices

\begin{tabular}{ccccccc}
\hline $\mathrm{ME}^{\mathrm{l}}$ & $\mathrm{CP}^{2}$ & Total Cost of Feed & Total Cost & Total Return & Net Profit & Economic Efficiency $^{\mathrm{l}}$ \\
\hline 2600 & 14 & $40.02 \pm 1.8^{\mathrm{a}}$ & $100.02 \pm 10.16^{\mathrm{a}}$ & $92.4 \pm 3.12^{\mathrm{b}}$ & $-7.62 \pm 0.64^{\mathrm{f}}$ & $-19 \pm 2.1^{\mathrm{f}}$ \\
& 16 & $41.4 \pm 2.41^{\mathrm{a}}$ & $101.4 \pm 6.45^{\mathrm{a}}$ & $95.6 \pm 5.35^{\mathrm{b}}$ & $-5.8 \pm 0.56^{\mathrm{e}}$ & $-14 \pm 2.4^{\mathrm{f}}$ \\
& 18 & $42.48 \pm 2.98^{\mathrm{a}}$ & $102.48 \pm 8.64^{\mathrm{a}}$ & $91.9 \pm 8.81^{\mathrm{bc}}$ & $-10.58 \pm 0.92^{\mathrm{g}}$ & $-24.9 \pm 2.1^{\mathrm{g}}$ \\
2800 & 14 & $36.52 \pm 3.16^{\mathrm{b}}$ & $93.52 \pm 6.41^{\mathrm{b}}$ & $92.5 \pm 4.14^{\mathrm{b}}$ & $-4.02 \pm 0.86^{\mathrm{e}}$ & $-11 \pm 1.8^{\mathrm{e}}$ \\
& 16 & $36.03 \pm 3.23^{\mathrm{b}}$ & $91.03 \pm 3.05^{\mathrm{b}}$ & $98.2 \pm 3.68^{\mathrm{b}}$ & $2.57 \pm 0.61^{\mathrm{c}}$ & $6 \pm 1.46^{\mathrm{c}}$ \\
& 18 & $38.09 \pm 5.2^{\mathrm{a}}$ & $92.09 \pm 4.24^{\mathrm{b}}$ & $98.4 \pm 3.27^{\mathrm{b}}$ & $0.71 \pm 0.17^{\mathrm{d}}$ & $0.8 \pm 3.1^{\mathrm{d}}$ \\
3000 & 14 & $32.62 \pm 4.13^{\mathrm{c}}$ & $88.62 \pm 3.99^{\mathrm{b}}$ & $95.7 \pm 6.54^{\mathrm{b}}$ & $7.08 \pm 0.42^{\mathrm{b}}$ & $21.7 \pm 3.8^{\mathrm{b}}$ \\
& 16 & $29.87 \pm 2.37^{\mathrm{d}}$ & $81.87 \pm 6.51^{\mathrm{c}}$ & $110.3 \pm 8.14^{\mathrm{a}}$ & $10.43 \pm 0.69^{\mathrm{a}}$ & $34.9 \pm 1.4^{\mathrm{a}}$ \\
$\mathrm{P}-\mathrm{values}$ & 18 & $33.62 \pm 2.79^{\mathrm{c}}$ & $93.62 \pm 5.48^{\mathrm{b}}$ & $90.8 \pm 5.14^{\mathrm{c}}$ & $-2.82 \pm 0.84^{\mathrm{e}}$ & $-8.4 \pm 1.2^{\mathrm{e}}$ \\
$\mathrm{ME}$ & & & & & & $<0.0001$ \\
$\mathrm{CP}$ & & $<0.0001$ & $<0.0001$ & $<0.0001$ & $<0.0001$ & $<0.0001$ \\
$\mathrm{ME} \times \mathrm{CP}$ & $<.096$ & 0.041 & 0.036 & $<0.0001$ & $<0.0001$
\end{tabular}

${ }^{1} \mathrm{ME}$ : metabolizable energy; ${ }^{2} \mathrm{CP}$ : crude protein. Means within the same column carrying different superscripts were significantly different at $\mathrm{P}<0.05$ based on Bonferroni multiple comparison test

${ }^{1}$ Economic efficiency $=$ Net return $/$ Price of feed cost.

\section{Discussion}

Considering our main interest was to establish a bio-economic optimum for feed formulation that meet the nutritional requirements of pigeons and maintain maximum performance with least cost as well.

\section{Growth Performance}

Body weights in different ages were a significantly improved, squabs fed the highest ME and CP level (16-18\% with 3000 Kcal $\mathrm{ME} / \mathrm{kg}$ ) of diet recorded significantly highest body weight and average daily gain contrast with other treatments. These results are in the line with others who pointed out that $15.5 \%$ protein and $16 \% \mathrm{CP}$ had a significant positive influence on average $\mathrm{BW}$ in growing squabs from 28 days to $6^{\text {th }}$ month of age $[5,10]$. While, these results disagreed with AbdelAzeem [11], who recommended that the best protein level of Baladi pigeons diets was $14 \%$ $\mathrm{CP}$ with $3100 \mathrm{ME} \mathrm{kcal} / \mathrm{kg}$ that produced the better performance (fertility, livability, squab production, the return of squabs and body measurements of squabs). Also, the body weight and weight gain were not significantly affected when dietary metabolic levels increased up to $3200 \mathrm{ME} \mathrm{Kcal} / \mathrm{kg}$ of pigeon diet [8].

In terms of the FI, increasing energy and CP level results in decreased feed intake. This indicated that birds fed more feed on the lowenergy diet to fulfill their energy requirements and normal growth when compared with those of high-energy diet. This result is in accordance with previous studies that reported the FI decreased significantly as ME increased from 2,650 to $3,150 \mathrm{Kcal} / \mathrm{kg}[8,12,13]$. Abdel-Azeem [11], showed that increase the level of protein diet (14, 17 and 20\%) of pigeons caused a decreased in the amount of feed consumed at different periods. However, the daily feed intake of pigeon increased when the protein in diet increased from 12 to $20 \%$ [6].

\section{Carcass traits}

The current results revealed significant increase in dressing, breast and thigh muscles percentage with increase energy and protein level. While, there was no significant difference in liver, heart, gizzard percentage among the treated groups. These are in agreement with those who reported that the muscle (breast and drumstick) yields were significantly higher in broiler fed high protein diet when compared with those of medium and low protein diets [20]. Aggoor et al. [21] found that increasing ME content of the control diet of quail with adding fat, produced carcasses of higher dressing percentage, whilst had no effect on gizzard, liver, giblets, heart and testis percentage. On the contrary, the carcass weight and yield of breast muscle remained unaffected by changes in dietary energy or lysine content in quail's diet [22]. 


\section{Biochemical analysis}

The knowledge of hematological parameters is deficient and there are only few reports on the parameters of blood analysis in pigeons. The current results indicated that the plasma protein and lipids fractions were affected by the nutritional status of birds. In our study, increased both dietary energy and protein level, elevated the plasma protein and lipid parameters. The previous findings are in agreement with those found by others, who reported that energy and protein levels in diet influenced the chicken plasma metabolites $[14,15]$. These results were in line with those of other researchers who recorded that the plasma total protein, globulin, urea, uric acid cholesterol and triglyceride [8,16,17]. High density lipoprotein and LDL were increased by increasing the dietary CP (14, 17 and 20\%) and metabolic energy level (2600, 2800, 3000 and $3200 \mathrm{ME} \mathrm{Kcal).} \mathrm{While,} \mathrm{these} \mathrm{results}$ disagree with Abdel-Azeem et al. [18] and Abou-Zeid et al. [19] who found that the total plasma protein, albumin and total cholesterol levels were not significantly affected by feeding Japanese quail on diet containing 20, 22 and $24 \% \mathrm{CP}$.

\section{Economic analysis}

The cost of feeding represents the largest item in the variable costs. Cost of feeding and total costs were significantly higher in groups fed on $2600 \mathrm{ME} \mathrm{Kcal} / \mathrm{kg}$ with different levels of protein, while the lower were in group fed on $3000 \mathrm{ME} \mathrm{Kcal} / \mathrm{kg}$ with $16 \%$ protein. Fixed costs are cost depreciation, labor, electricity, equipment and building. The fixed costs represented a minor proportion of the total costs while the total variable costs represented the major portion of the total costs. The revenues of pigeon squab's pair included sales of squabs as layers. Net profit and economic efficiency were significantly higher in group fed on $3000 \mathrm{ME} \mathrm{Kcal} / \mathrm{kg}$ with $16 \%$ protein followed by group fed on $3000 \mathrm{ME} \mathrm{Kcal} / \mathrm{kg}$ with $14 \%$ protein. These findings are strongly confirmed the findings of Khashaba et al. [10], who detected that the highest economic efficiency was in group fed on dietary protein level $16 \% \mathrm{CP}$ with $2900 \mathrm{ME} \mathrm{Kcal} / \mathrm{kg}$. Quails diet contained high energy $(3100 \mathrm{Kcal} / \mathrm{Kg})$ was enhanced the production efficiency with least cost [22].

\section{Conclusion}

The metabolizable energy and crude protein content of pigeon's diets play important roles on performance of pigeon. Feeding high crude protein (16\%) and metabolizable energy (3000 $\mathrm{ME} \mathrm{Kcal} / \mathrm{kg}$ ) diets improved pigeon squab's body weight, carcass characteristics, serum biochemical analysis and economic efficiency. It is recommended to formulate the pigeon diet containing high energy $(3000 \mathrm{Kcal} / \mathrm{Kg})$ with $16 \%$ crude protein to maximize the production with least cost.

\section{Acknowledgment}

The authors would like to appreciate and thank Dr. Ashraf Hussein, Department of Avian and Rabbit Medicine, for providing information and guidance about pigeon breeding and management. Dr. Mohamed ElSayed, Department of Nutrition and Clinical Nutrition, for helping in ration formulation. Dr. Mohamed Afifi, Animal Wealth Development Department, for his assistance in statistical analysis.

\section{Conflict of interest}

The authors declare that they have no conflict of interest.

\section{References}

[1] Sales, J. and Janssens, G. (2003): Nutrition of the domestic pigeon (Columba liviadomestica). World Poult Sci J 59: 221232.

[2] Pomianowski, J.F.; Mikulski, D.; Pudyszak, K.; Cooper, R.G.; Angowski, M.; Joźwik, A. and Horbańczuk, J.O. (2009): Chemical composition, cholesterol content, and fatty acid profile of pigeon meat as influenced by meat-type breeds. Poult Sci, 88(6): 1306-1309.

[3] Mohamed, W.A. (2016): Studies on improving productivity of local pigeons under the Egyptian conditions. Master Thesis, Department of Animal Production Faculty of Agriculture Al-Azhar University.

[4] National Research Council (NRC), (1994): Nutrient Requirements of Poultry. $9^{\text {th }}$ recevised edition. Nat, Acad Press, Washington D.C. 
[5] Bu, Z.; Xie, P.; Fu, S.Y.; Tong, H.B. and Dai, X. (2015): Effect of energy and protein levels on performance, egg quality, and nutrient digestibility of laying pigeons. J Appl Poult Res, 24(3): 371-379.

[6] Meleg, I.; Dublecz, K.K.; Vincze, L. and Horn, P. (1999): Effect of dietary crude protein level on reproductive traits of commercial pigeons in different production terms. Acta Agraria Kaposvariensis, 3: 247-253.

[7] Costantini, D. (2010): Complex trade-offs in the pigeon (Columba livia): Egg antioxidant capacity and female serum oxidative status in relation to diet quality. Comp. Biochem Phys Part B, 180: 731739.

[8] Abdel-Azeem, F.A.; El-shafei, A.A. and Abdullah, E.A. (2007): Studies on the effect of different dietary metabolizabole energy levels on some performance of local Baladi pigeons. Egypt Poult Sci, 27(3): 595-611.

[9] Abdel-Azeem, F.A. and El-Shafei, A.A. (2006): The pigeon (Columba Livia Domestica): A Review. Egypt Poult Sci, 26(3): 1153-1168.

[10] Khashaba, H.A; Sayed, M.A.M; Mariey, Y.A. and Ibrahim, M.A. (2009): Nutritional and management studies on the pigeon: Estimate of metabolizabol energy requirements. Egypt Poult Sci, 29(2): 481-501.

[11] Abdel-Azeem, F. (1998): Effect of protein level on some nutritional and physiological parameters in pigeon. $\mathrm{PhD}$ thesis, Agric Al-Azhar University.

[12] Waldie, G. A.; Olomu, J.M.; Cheng, K.M. and sim, J. (1991): Effect of two feeding system, two protein levels and different dietary energy sources and level of performance of squabbling pigeons. Poult Sci. 70:1260-1212.

[13] Khashaba, H.A.; Mariey, Y.A. and Ibrahem, M.A. (2010): Nutritional and management studies on the pigeon: effect of dietary 1-carnitine supplementation with different energy levels on productive and reproductive performance of pigeon. Egypt Poult Sci, 30(I): 1-23.

[14] Caldera, R.M.; Bed, A.T.; Santos, C.C.; Vazquez, M.I. and Portugal, A.V. (2007): The effect of body condition score on blood metabolites and hormonal profiles in ewes. Small Rumin Res, 68: 233-241.

[15] Teteh, K.T.; Aklikokou, K.; Gbeassor, M.; Buyse, J. and E. Decuypere (2010): Effect of low-protein or high energy levels diets on layer-type chick juvenile performance. Intr J Poult Sci, 9(12): 1156-1160.

[16] Zeweil, H.S.; Abdalah, A.A.; Ahmed, M.H. and Marwa, R.S.A. (2011): Effect of different levels of protein and methionine on performance of baheij laying hens and environmental pollution. Egypt Poult Sci, 31: 621-639.

[17] Abdel-Azeem, F.A. (2011): Influence of qualitative feed restriction on reproductive performance of Japanese quail hens. Egypt Poult Sci, 31(4): 883897.

[18] Abdel-Azeem, F.; Faten, A.A.I. and Nematallah, G.M.A. (2001): Growth performance and some blood parameters of growing Japanese quail as influenced by dietary different protein levels and microbial probiotics supplementation. Egypt Poult Sci, 21(2): 465-489.

[19]Abou-Zeid, A.A.; Gaber, S.; El-Samra AbouEgla and Zeweil, H.S. (2000): Effect of dietary protein level and NFAC 1000 supplementation on performance, digestibility and carcass in growing Japanese quail. J Agriculture Sci, 25(2): 729-738.

[20] Laudadio, V.; Passantino, L.; Perillo, A.; Lopresti, G.; Passantino, A.; Khan, R.U. and Tufarelli, V. (2012): Productive performance and histological features of intestinal mucosa of broiler chickens fed different dietary protein levels. Poult Sci, 91(1): 265-270.

[21] Aggoor, F.A.M.; Attia, Y.A. and Qota, E.M.A. (2000): A study on the energetic efficiency of different fat sources and 
levels in broiler chick vegetable diets. J Agric Sci, 25: 801-820.

[22] Mubarik, M.; Abdur Rahman, S.; Muhammad, A.; Talat, N.P. and
Makhdoom, A.J. (2014): effect of dietary energy levels on growth performance and feed cost analysis in Japanese quail. Pak J Zool, 46(5): 1357-1362.

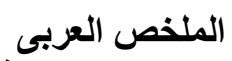

$$
\begin{aligned}
& \text { التقييم الإقتصادي لبعض الممارسات الثذائية فى زغاليل الحمام }
\end{aligned}
$$

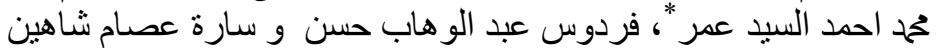

$$
\begin{aligned}
& \text { قسم تتمية الثروة الحيو انية - كلية الطب البيطرى - جامعة الزقازيق }
\end{aligned}
$$

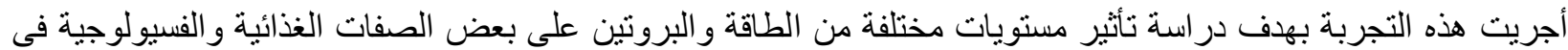

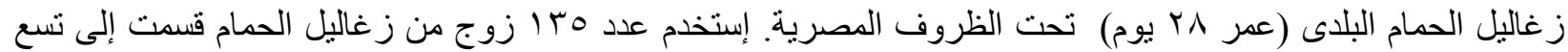

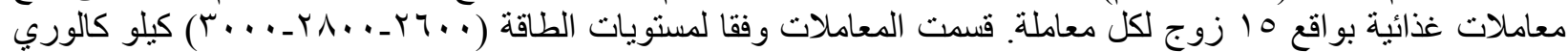

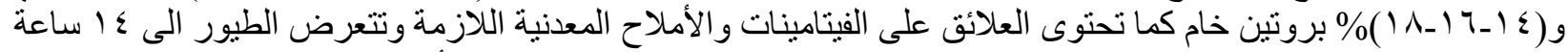

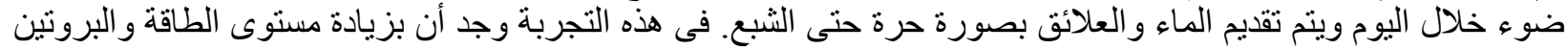

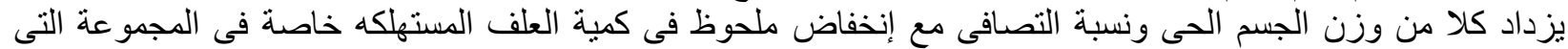

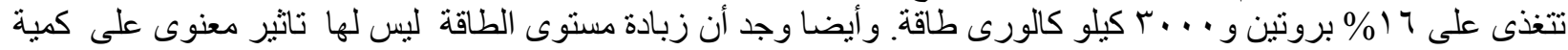

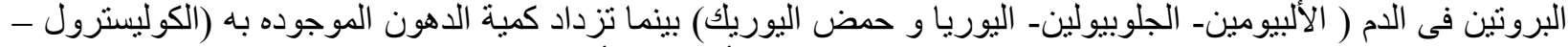

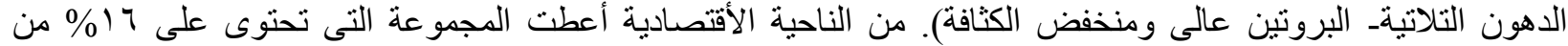

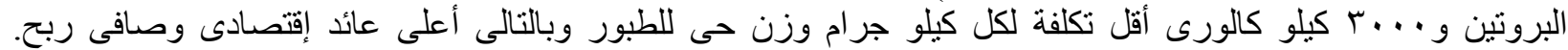

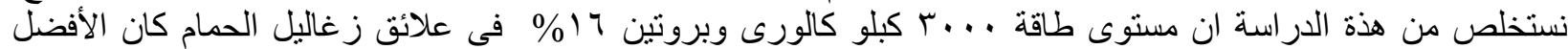
للحصول على أعلى أداء إنتاجى بتكاليف أقل وبالتالى زيادة الكفاءة الاقتصادية. 